\title{
Total talar replacement with a novel 3D printed modular prosthesis for tumors
}

This article was published in the following Dove Press journal:

Therapeutics and Clinical Risk Management

Xiang Fang'
Hongyuan Liu'
Yan Xiong'
Wenli Zhang'
Yi Luo'
Fan Wu'
Yong Zhou'
Liuhong Song
Zeping Yu'
Chongqi Tu'
Hong Duan'
'Department of Orthopedics, West
China School of Medicine/West China
Hospital, Sichuan University, Chengdu,
Sichuan, People's Republic of China;
${ }^{2}$ Department of Orthopedics, Fourth
People's Hospital of ZiGong, ZiGong,
Sichuan, People's Republic of China;
${ }^{3}$ Department of Orthopedics, People's
Hospital of Pengzhou, Pengzhou,
Sichuan, People's Republic of China

Correspondence: Hong Duan

Department of Orthopedics, West China School of Medicine/West China Hospital, Sichuan University, 37 Guo Xue Lane, Chengdu, Sichuan 610064, People's

Republic of China

Tel/fax +86 2885422578

Email duanhong1970@I26.com
Purpose: Arthrodesis is one of the most widely accepted surgical recommended methods for tumors of the talus, but it may be associated with poor limb functions. The aim of this study was to present a novel reconstruction with ankle function preserved after en bloc talus tumor resection.

Patient and method: A 43-year-old female with mesenchymal sarcoma of the talus was admitted in West China Hospital. Total talar replacement with three-dimensional (3D) printed modular prosthesis was prepared for reconstruction. The 3D printed modular prosthesis was designed exactly as the mirror image of the contralateral talus with complete filling of the sinus tarsi and subtalar joint space. The upper modular component of prosthesis was made of ultra high molecular weight polyethylene, and the lower component, titanium alloy. Pre-drilled holes in three directions were prepared for screw fixation of the subtalar joint.

Results: The patient underwent en bloc talus resection through anterior approach, followed by reconstruction with the $3 \mathrm{D}$ printed prosthesis. The whole procedure took 2 hours, and intra-operative blood loss was $50 \mathrm{~mL}$. At the last follow-up, our patient was disease free and she could walk almost normally without any aid or pain. The Musculoskeletal Tumor Society score was 26/30. The American Orthopedic Foot and Ankle Society score was 91/100. The range of motion for dorsiflexion and plantar flexion was $40^{\circ}$. And no abnormalities were observed in the roentgenograph. Conclusion: Total talar replacement with a 3D printed modular prosthesis may be an effective procedure for patients with tumors of the talus as it could maintain ankle function.

Keywords: bone tumors, sarcoma, reconstructive surgery, ultra high molecular weight polyethylene, limb salvage, en bloc resection

\section{Introduction}

Malignant tumors around the foot and ankle are rare, accounting for less than $1 \%$ of all malignancies. ${ }^{1,2}$ Approximately $22 \%-39.2 \%$ of these tumors are malignant. ${ }^{3,4}$ Metatarsals and calcaneum are the most common location, followed by the phalanges and talar. ${ }^{2}$ Though ankle arthrodesis is one of the most widely accepted surgical reconstruction method for talus tumors, consequent poor ankle function cannot be ignored.

Tumor-induced bone defects can be reconstructed with three-dimensional (3D) printing technology accurately and anatomically by tailored contour, which can induce bone ingrowth through the porously proofing treatment, reduce long-term mechanical complication, and promote better function of limbs. ${ }^{5}$ However, no similar report has been retrieved regarding talus prosthesis for tumors so far. As a result, we present a case of a 43-year-old female with mesenchymal sarcoma of the talus treated with total talar replacement with a novel 3D printed custom-made modular prosthesis. The aim of this study was to describe the design of the prosthesis for total talar replacement, the surgical technique, and the clinical outcome of the patient. 


\section{Patient and methods}

\section{Case report}

A 43-year-old female was admitted to our outpatient clinic with a 6-month history of progressive right ankle swelling and pain during walking. Prior history regarding trauma or infection, family history of tumor, metabolic and rheumatic conditions was negative. Physical examination indicated swelling ankle with mild tenderness and slightly restricted range of motion. The Musculoskeletal Tumor Society (MSTS) score was 19/30. ${ }^{6}$ The American Orthopedic Foot and Ankle Society (AOFAS) score was $73 / 100 .^{7}$ For the affected and the contralateral ankle, respectively, the dorsiflexion was $15^{\circ}$ and $20^{\circ}$; the plantar flexion was $45^{\circ}$ and $55^{\circ}$; the eversion was $25^{\circ}$ and $35^{\circ}$; and the inversion was $25^{\circ}$ and $30^{\circ}$. Roentgenograph showed a gross osteolytic bone destruction in the talus (Figure 1A). Computed tomography scan revealed an extensive lesion with cortical breaks in the talus (Figure 1B). MRI showed massive involvement of the talus with intact calcaneum, navicular, distal tibia, and distal fibula (Figure 1C). Bone scan revealed an increased uptake around the ankle (Figure 1D). Biopsy showed a mesenchymal tumor of malignancy or borderline malignancy. And there was no metastasis in the staging study. Since the talus was almost completely involved, we planned to perform a total talar replacement through anterior approach with 3D-printed prosthesis.

Written informed consent was provided by the patient to participate in this study including permission for images and medical data to appear in print and online.

\section{Design and manufacture of prosthesis}

A press-fit modular prosthesis was designed according to the mirror image from CT scan of the contralateral talus, with complete filling of the sinus tarsi and subtalar joint space. The upper modular component of the prosthesis was made of ultra high molecular weight polyethylene (UHMWPE) for articulation with the tibia and fibula, and the lower component, titanium alloy for articulation with the calcaneum and navicular (Figure 2). The modular design had three distinct benefits: (1) it could facilitate optimal screw fixation; (2) the modular components could be made of different materials for different purposes; (3) if the UHMWPE component was worn, it could be easily replaced and revised. As for the lower component, screw holes in three directions were predrilled to fix the calcaneum, and porous structures were prepared to further promote subtalar arthrodesis. The prosthesis was designed by Creo Parametric 2.0 (PTC, Boston, MA, USA)
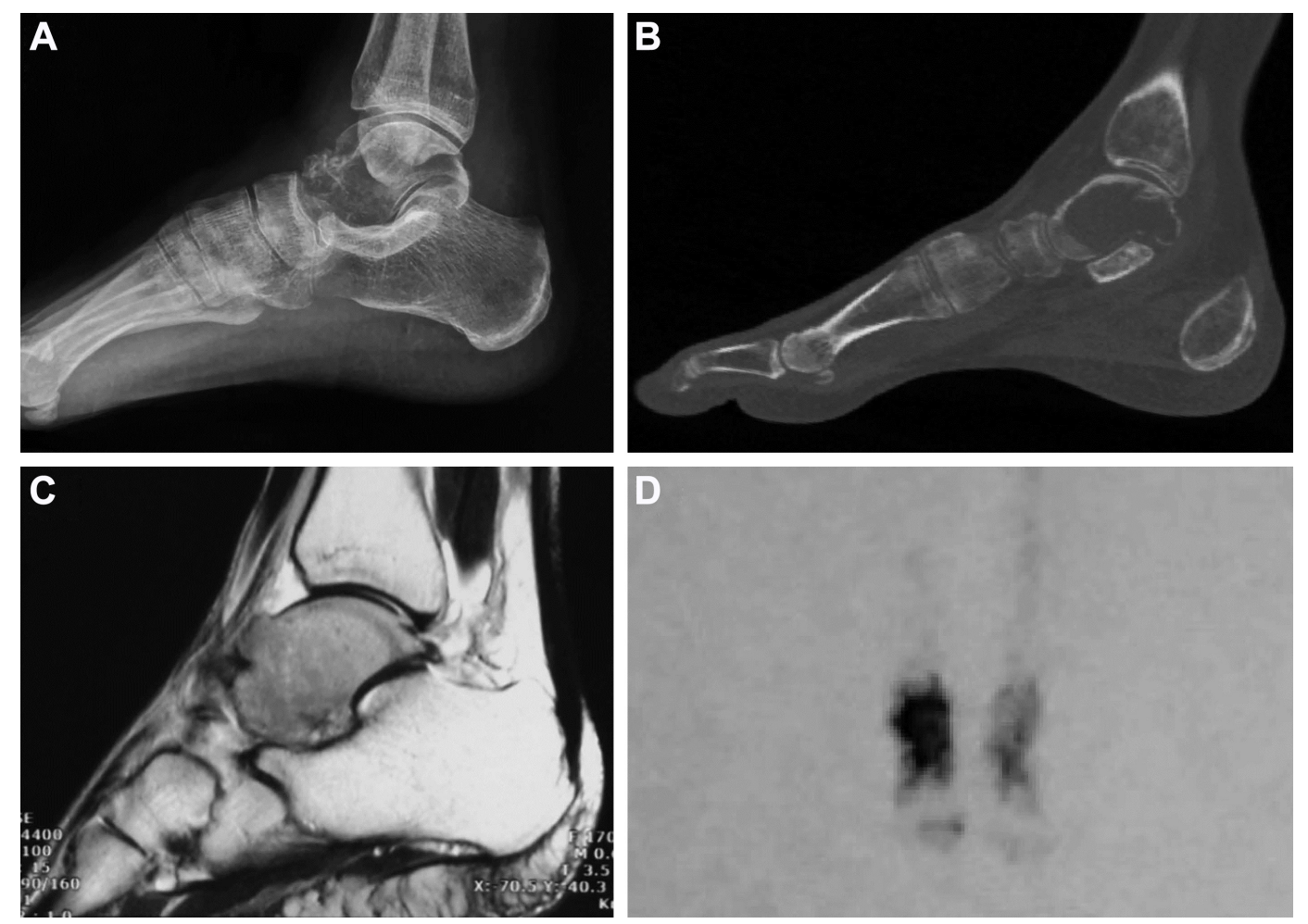

Figure I The preoperative images.

Notes: (A-C) An extensive osteolytic lesion in the talus. (D) Bone scan revealed an increased uptake around the ankle. 


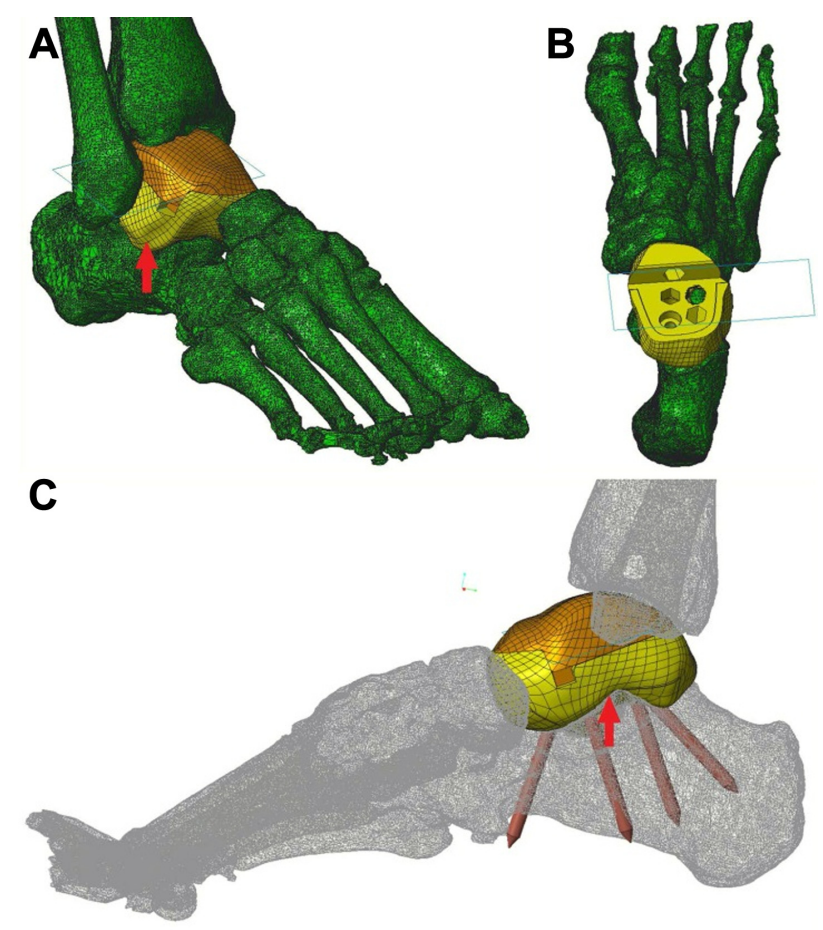

Figure 2 The prosthesis design.

Notes: (A) Anterolateral view of the installed prosthesis, with the sinus tarsi completely filled (red arrow). (B) Top view of the lower modular component of the prosthesis placed on the calcaneus. Pre-drilled holes for screws can be seen. (C) Mediolateral view of the installed prosthesis showing the direction of the screw fixation (not the length of screws). The sinus tarsi is completely filled (red arrow). and manufactured by ChunLi Co. (Beijing, People's Republic of China) with 3D printing technique. The diameter of pores was $600 \mu \mathrm{m}$, with an average porosity of $50 \%-80 \%$. And a model of the prosthesis was printed and tested before the final production to verify our plan (Figure 3).

\section{Results}

\section{Surgical procedure}

The patient was in supine position with affected thigh elevated and pressurized by tourniquet. During surgery, the talus was exposed through anterior approach between the extensor digitorum longus and the extensor hallucis longus tendons. The medial branch of superficial fibular nerve and anterior tibial artery were identified and well protected. After the dissection of the soft tissues, the talus was en bloc resected. Then the subtalar cartilage of the calcaneus was removed to facilitate arthrodesis, and the lower modular component of the prosthesis was inserted and fitted to the calcaneus and navicular. Four screws in three directions were placed to fix the subtalar joint through the pre-drilled holes in the prosthesis. Then the upper modular component was pressed in and fitted to the lower component. The remaining capsule was then repaired as much as possible. During the whole

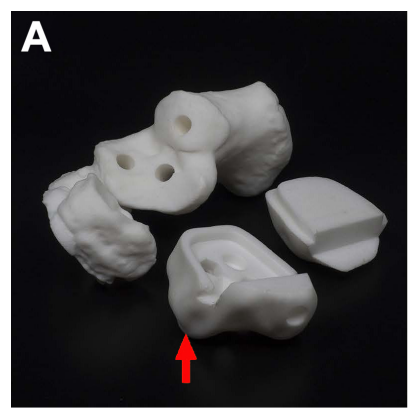

D

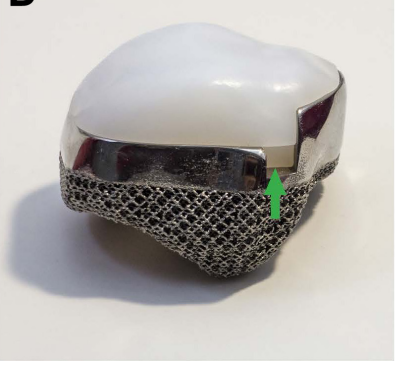

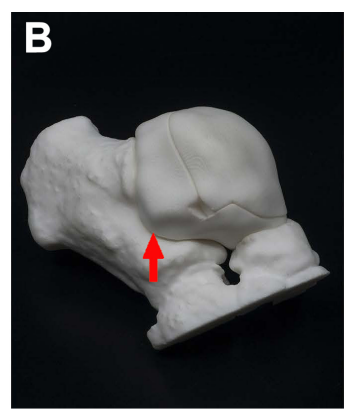

E

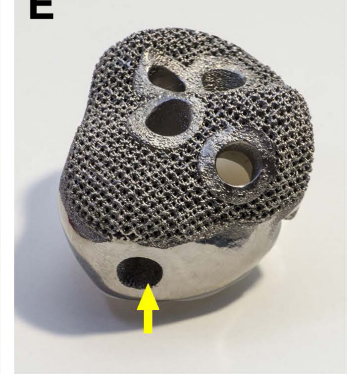

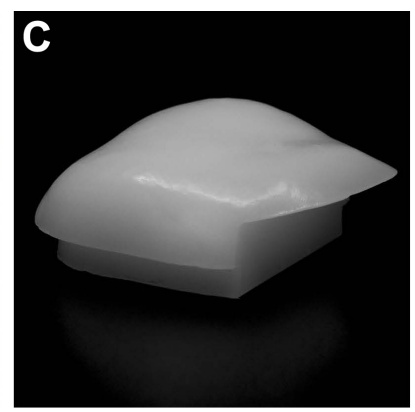

$\mathbf{F}$

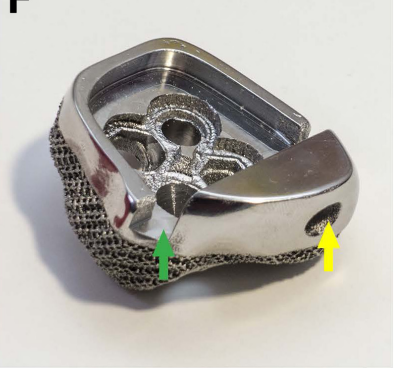

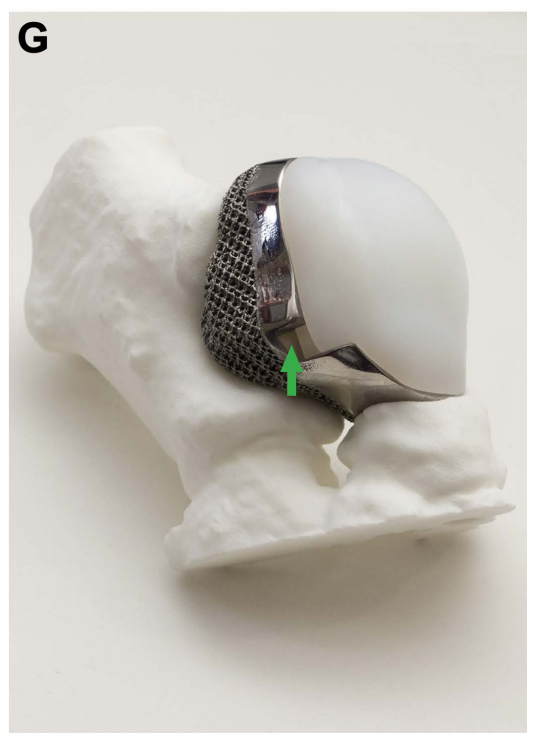

Figure 3 The models and the finished product.

Notes: (A, B) Models of the prosthesis and adjacent bones were printed and tested to verify our plan before the final production. Anterolateral view of the installed prosthesis, with the sinus tarsi completely filled (red arrow). (C-F) The three-dimensional printed press-fit modular prosthesis. The lower modular component (E, F), with porous structures on the bottom to facilitate subtalar arthrodesis, is made of titanium alloy. The upper modular component (C) is made of ultra high molecular weight polyethylene (UHMWPE). (G) The installed prosthesis fits well to the calcaneus and navicular bone. The gap (green arrow) is designed for easy separation of the two modular components. The hole (yellow arrow) is predrilled for fixation of the talonavicular joint, but it was not fixed during the surgery in order to obtain better foot function. 

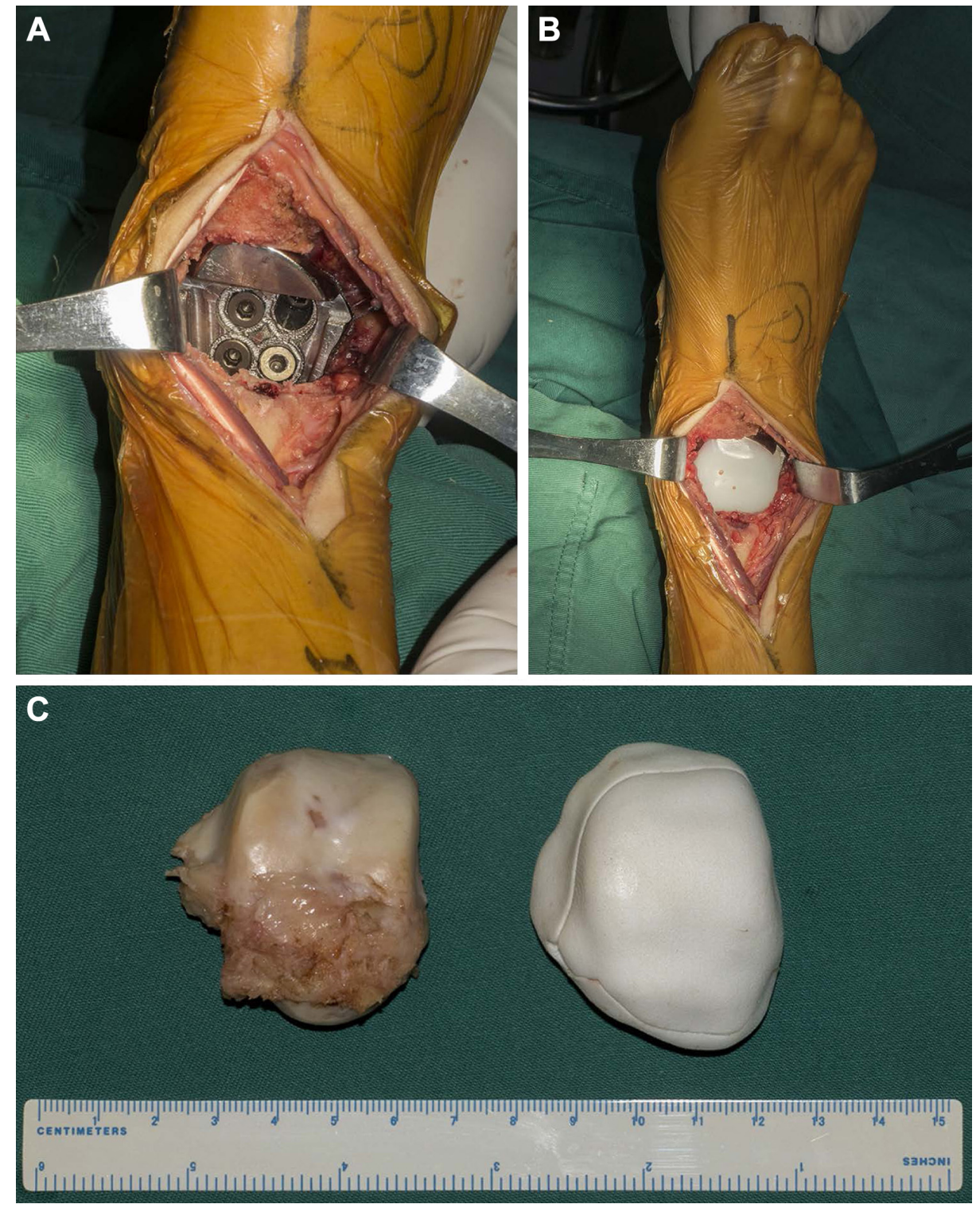

Figure 4 The intraoperative pictures.

Notes: (A, B) After the removal of the talus through anterior approach, the modular prosthesis was inserted and fixed to the calcaneus. (C) The en bloc resected talus was almost identical to the three-dimensional printed model of the prosthesis of the talus.

procedure, the superficial layer of the deltoid ligament, the calcaneofibular ligament, and the ankle cartilage were preserved intact, and no ligament reconstruction was performed (Figure 4). The whole procedure took 2 hours, and intraoperative blood loss was $50 \mathrm{~mL}$. Postoperative roentgenograph showed the implant was in good position (Figure 5).

\section{Histological findings and postoperative management}

Post pathological examination revealed mesenchymal sarcoma, the Fédération Nationale des Centers de Lutte Contre le Cancer grade 2. And the margins were clear. In the postoperative period, the drainage tube was kept for 3 days, and
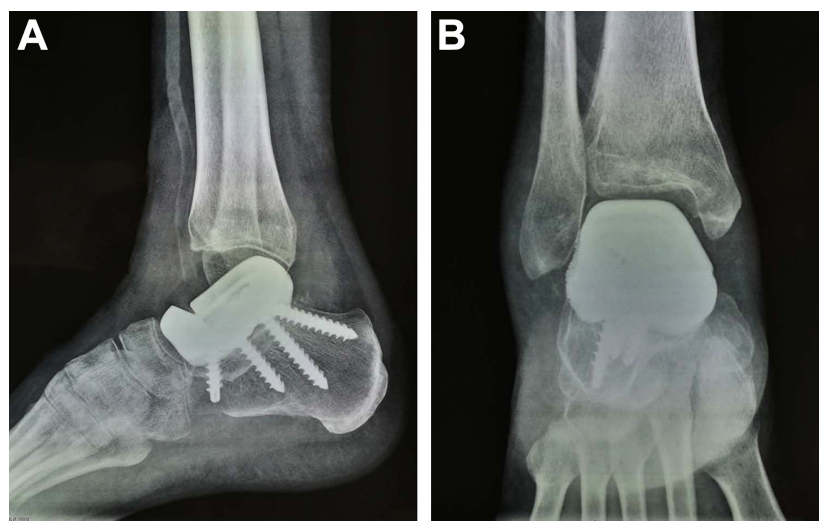

Figure $\mathbf{5}$ The postoperative roentgenographs.

Note: (A, B) The modular prosthesis and the screws are in good position. 

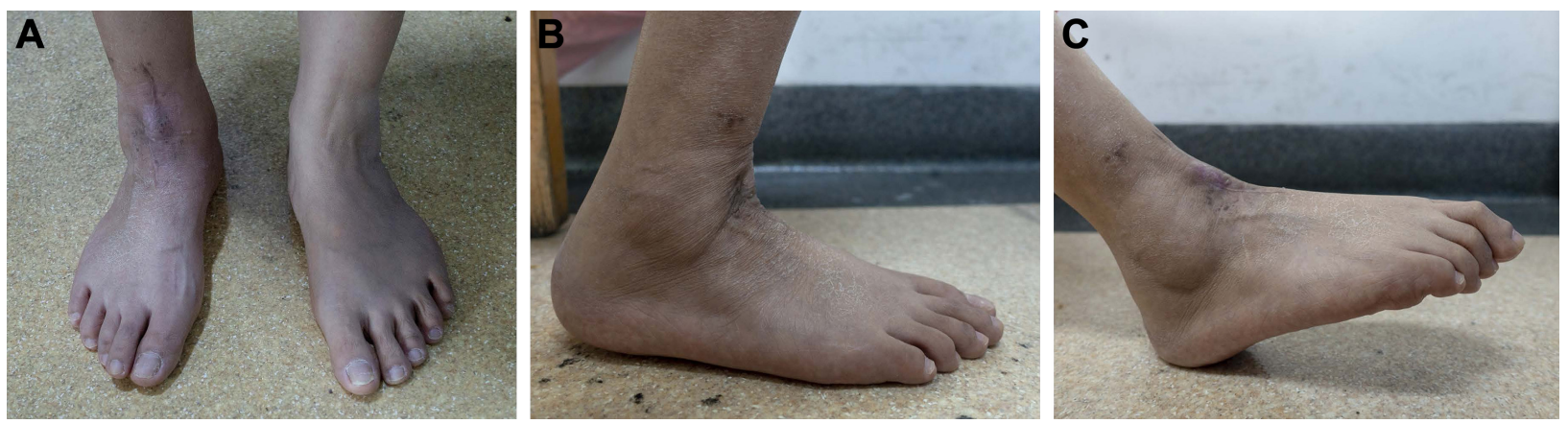

Figure 6 Standing appearance.

Note: (A) Dorsiflexion/plantarflexion (B, C) around 6 months after total talar replacement with the three-dimensional printed modular prosthesis in the right foot.

intravenous antibiotics were administered for 5 days. Cast or orthosis was not used during the postoperative period. Range of motion exercises started 2 weeks after surgery, and gradually increased weight bearing was initiated 4 weeks postoperatively. However, our patient suffered from delayed wound healing near the extensor hallucis longus, and the wound healed nonoperatively 3 months after surgery. This prevented her from doing functional exercises as we had planned. There were no other perioperative complications. Our patient also received four cycles of chemotherapy one month postoperatively.

\section{Follow-up}

At the last follow-up, 6 months after surgery, our patient was disease free, and she could walk almost normally without any aid or pain. The MSTS score was 26/30. The AOFAS score was 91/100. For the affected and the contralateral ankle, respectively, the dorsiflexion was $10^{\circ}$ and $20^{\circ}$; the plantar flexion was $30^{\circ}$ and $55^{\circ}$; the eversion was $5^{\circ}$ and $35^{\circ}$; the inversion was $10^{\circ}$ and $30^{\circ}$ (Figure 6). Roentgenograph showed that the prosthesis and the screws were in stable position, and no abnormalities were observed in the surrounding bones (Figure 7).

\section{Discussion}

The surgical management of malignancy of the talus is difficult to achieve both adequate surgical margins and a functional reconstruction. Widely accepted operative reconstruction options for talus tumors include talocrural arthrodesis and shortening arthrodesis between the calcaneus and the tibia. ${ }^{8,9}$ However, these procedures may be associated with long recovery periods. In addition, the range of motion of the ankle joint is completely sacrificed.

Total talar replacement with a prosthesis, first performed by Taniguchi et al in 2005, is considered to be an optimal treatment for extensive aseptic necrosis of the talus, and traumatic loss of talus. ${ }^{10}$ Preservation of the joint motion and shorter recovery are the major advantages over traditional surgical options. And we believe it would also be of significance in treating talus tumors. We reviewed all causes for total talar replacement and summarized eight articles with a total of 74 taluses replaced (Table 1). ${ }^{10-17}$ Those studies regarding distal tibia replacement were excluded, except revision with total talar replacement after ankle arthroplasty. The most common reason for performing total talar replacement was osteonecrosis of the talus, followed by trauma. And no tumor related total talar replacement was ever reported. As for the design of the prosthesis, they were anatomic and unibody and made of metal or aluminum-ceramic. And in general, most of these patients had a satisfactory outcome during follow-up.

Currently, the 3D printing technology, featured with rapid manufacture and precision, has presented new concepts in clinical practice, especially in orthopedics and plastic surgery. It has been successfully adopted in the reconstruction for bone defect on the sternum, limbs, pelvis, and upper
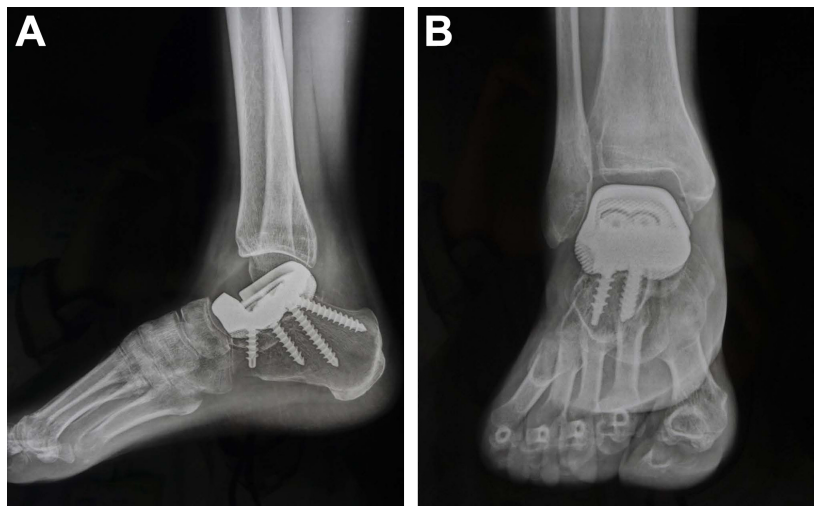

Figure 7 Follow-up radiographs 6 months after surgery.

Note: (A, B) The prosthesis is well positioned, and no abnormalities are noted. 


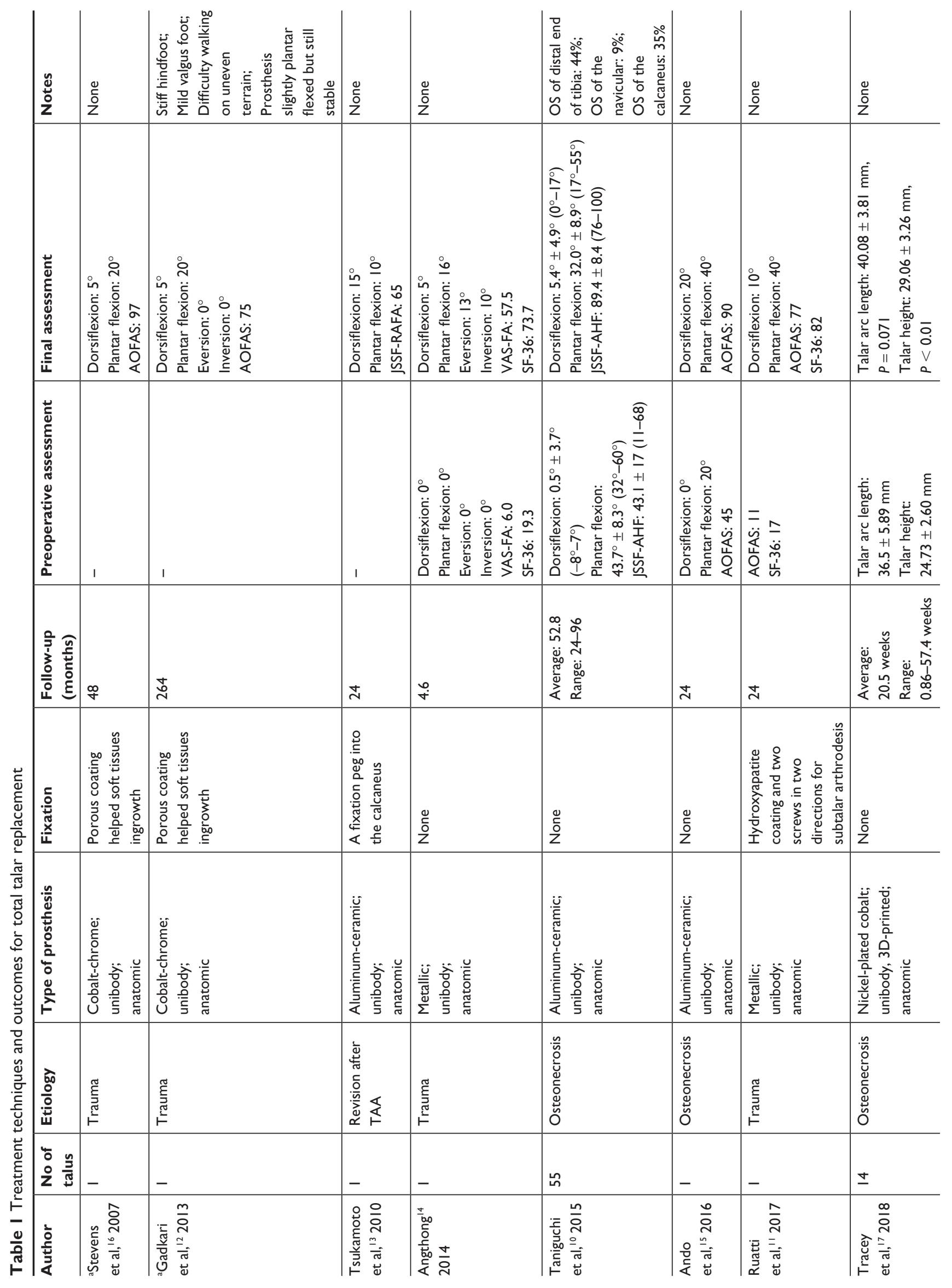




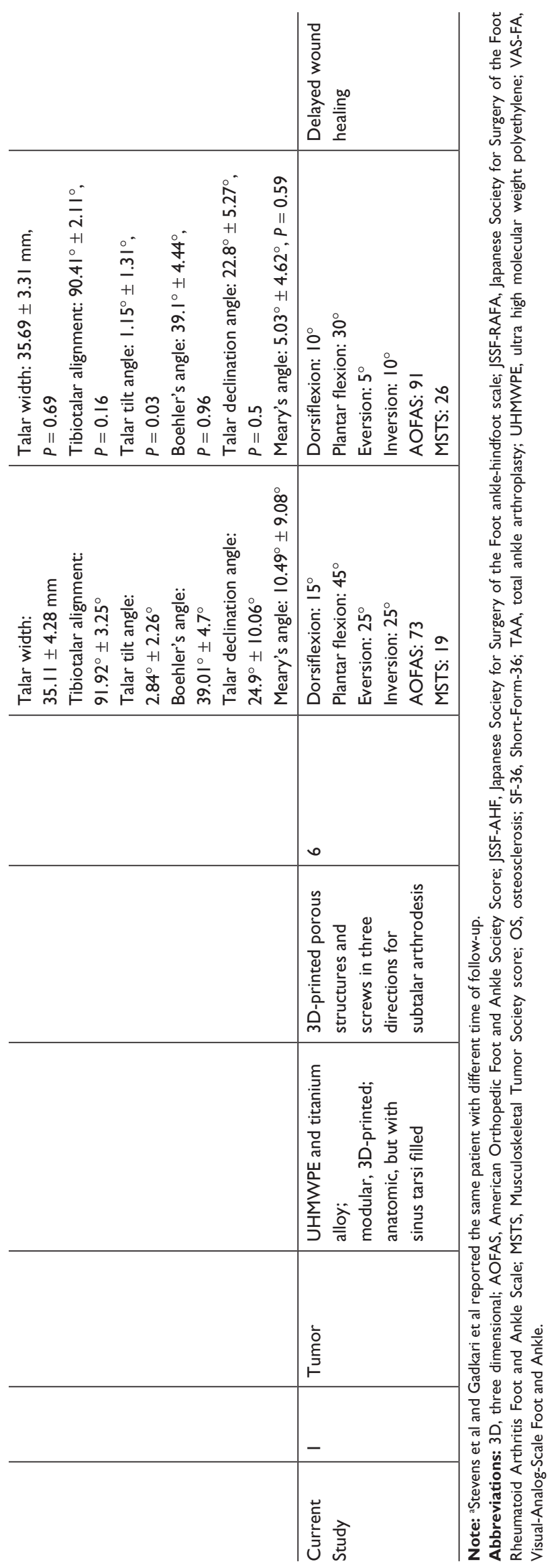

cervical spine. ${ }^{18-21}$ In our case, by 3D printing technique, a custom-made total talar prosthesis was produced completely based on the patient's own anatomic geometrics, which would contribute to better conformity of the articulation than those produced in traditional manufacture methods as were mostly reported in literature. Moreover, our modular design facilitating screws fixation in three directions, 3D-printed porous structures conducive for bone ingrowth, ${ }^{5}$ and the filling of the sinus tarsi would enable a firm purchase into the calcaneus, which indicates better initial stabilization, earlier functional exercise, and faster recovery than the previous prostheses used. Therefore, wear, the major barrier for prosthesis longevity, which is associated with conformity and constraint of the prosthesis-bone contact surface, ${ }^{22,23}$ may be greatly reduced theoretically in the long run. In the study by Taniguchi et al, ${ }^{10} 55$ ankles in 51 patients underwent total talar replacement with an aluminum-ceramic prosthesis. During an average of 52.8 months follow-up, 44\% of the distal tibia and $35 \%$ of the calcaneus had osteosclerosis in the radiographic assessment. The osteosclerosis may have resulted from the unmatched elastic modulus between the aluminum-ceramic material and the bone, and the bones were slightly worn by the high elastic modulus prosthesis material, which may lead to subsequent pain, instability, loosening of the prosthesis, and even revision, if this continued. In the current study, our prosthesis was modular design and made of two different materials. The upper part for articulation with the tibia, was made of UHMWPE, a common material used in hip prosthesis. Since UHMWPE has a relatively lower but closer elastic modulus with the bone, the wear, as well as the revision might be postponed. And even if the revision is inevitable, the UHMWPE modular component can be easily replaced due to the press-fit modular design.

When it comes to the filling of the sinus tarsi, no relevant literature has been reported for the total talar replacement. However, subtalar joint arthrodesis by placing an implant in the sinus tarsi, is a common surgical procedure performed in treatment for flexible flatfoot. Martinelli et al reported 49 pediatric patients treated with insertion of a permanent implant in the sinus tarsi. ${ }^{24}$ A total of $91.8 \%$ of these patients returned to normal sports activities after surgery. Needleman treated 28 feet of 23 adult patients with subtalar arthrodesis by sinus tarsi implant, and the occurrence of sinus tarsi discomfort was as high as $42 \%$ after implantation. ${ }^{25} \mathrm{Nev}-$ ertheless, Saxena et al performed a prospective study on 100 patients with adult acquired flatfoot deformity. ${ }^{26}$ The incidence of implant removal for discomfort was $22.1 \%$, but it was considered to be associated with inappropriate 
implant size. Our patient didn't develop any pain in foot during the 6-month follow-up. We speculated this may be a benefit of the 3D-printing technique, by which the contour of the prosthesis can be precisely adapted to the superior surface of calcaneus.

Although the subtalar joint arthrodesis might lead to limited ankle function, the functional outcome is acceptable. In the study by Yavuz et al, 20 patients (21 feet) with symptomatic subtalar arthrosis underwent isolated subtalar fusion. ${ }^{27}$ The mean postoperative AOFAS score improved from 61.7 (40-67) to 84.2 (65-94) during a mean follow-up of 43 (21-83) months. Ruatti et al performed a total talar replacement on a patient with traumatic loss of talus. ${ }^{11}$ Subtalar arthrodesis was performed without filling of sinus tarsi, and they yielded almost the same functional outcome as those without subtalar arthrodesis during the 2-year follow-up. And the range of motion of our patient was similar. As for sequela of isolated subtalar arthrodesis, the rate of tibiotalar and rear-foot osteoarthritis was $73 \%$ and $58.3 \%$, respectively, 10 more years after surgery, although the pain was well tolerated. ${ }^{28}$ The secondary osteoarthritis may result from excessive mechanical loading, which might accelerate the wear of the UHMWPE component. However, the osteoarthritis would thus be postponed.

For limitations, we failed to compare the cost regarding $3 \mathrm{D}$ printed prosthesis with other conventional total talus replacement prosthesis, since none of the literatures mentioned the cost. ${ }^{10-17}$ However, to facilitate future comparison, we listed our cost here. The $3 \mathrm{D}$ printed talus prosthesis, made by ChunLi Co., cost about 31,000 RMB (4,750 USD). And as a comparison, total ankle replacement prosthesis Inbone II (Wright Medical Technology, Memphis, TN, USA), cost about 90,000 RMB (13,760 USD) in our hospital. Another limitation is that our report has only one case with 6 months follow-up. Long-term follow-up is needed to further investigate the oncological and surgical outcome regarding this novel prosthesis. Nevertheless, we propose that it could be an alternative option for surgical reconstruction after total talar resection for all causes, including malignancy, osteonecrosis and traumatic loss of talus, as well as benign tumors of the talus with extensive involvement. And we will continue to follow our patient up.

\section{Conclusion}

Total talar replacement with 3D printed modular prosthesis may be a useful procedure for patients with extensive tumor involvement of the talus. The firm subtalar arthrodesis, the replaceable UHMWPE modular component, and the favorable congruency of the $3 \mathrm{D}$ printed prosthesis with adjacent joints might achieve long-term ankle function and stability.

\section{Acknowledgments}

This work was funded by the National Key Research and Development Program of China (No 2016YFC1102003) and the Science and Technology Research Program of Sichuan Province (No 2017SZ0095). No benefits in any form have been or will be received from a commercial party related directly or indirectly to the subject of this manuscript. And special thanks to Arun Rai from West China School of Medicine/West China Hospital, Sichuan University, China, for the language assistance regarding this study. West China Hospital, Sichuan University holds the patent for this modular total talar prosthesis.

\section{Disclosure}

Dr Duan, Dr Tu, Dr Fang, Dr Xiong, Dr Zhang, Dr Luo, and Dr Liu are inventors for this modular total talar prosthesis. The authors report no other conflicts of interest for this work.

\section{References}

1. Berlin SJ. A laboratory review of 67,000 foot tumors and lesions. J Am Podiatry Assoc. 1984;74(7):341-347.

2. Wu KK. Tumor review: osteogenic sarcoma of the foot. J Foot Surg. 1987;26(3):269.

3. Azevedo CP, Casanova JM, Guerra MG, Santos AL, Portela MI, Tavares PF. Tumors of the foot and ankle: a single-institution experience. J Foot Ankle Surg. 2013;52(2):147-152.

4. Chou LB, Ho YY, Malawer MM. Tumors of the foot and ankle: experience with 153 cases. Foot Ankle Int. 2009;30(9):836-841.

5. Shah FA, Snis A, Matic A, Thomsen P, Palmquist A. 3D printed Ti6Al4V implant surface promotes bone maturation and retains a higher density of less aged osteocytes at the bone-implant interface. Acta Biomater. 2016;30:357-367.

6. Enneking WF, Dunham W, Gebhardt MC, Malawar M, Pritchard DJ. A system for the functional evaluation of reconstructive procedures after surgical treatment of tumors of the musculoskeletal system. Clin Orthop Relat Res. 1993;286(286):241-246.

7. Kitaoka HB, Alexander IJ, Adelaar RS, Nunley JA, Myerson MS, Sanders M. Clinical rating systems for the ankle-hindfoot, midfoot, hallux, and lesser toes. Foot Ankle Int. 1994;15(7):349-353.

8. Blair HC. Comminuted fractures and fracture dislocations of the body of the astragalus. Am J Surg. 1943;59(1):37-43.

9. Dennison MG, Pool RD, Simonis RB, Singh BS. Tibiocalcaneal fusion for avascular necrosis of the talus. J Bone Joint Surg Br. 2001; 83(2):199-203.

10. Taniguchi A, Takakura $Y$, Tanaka $Y$, et al. An alumina ceramic total talar prosthesis for osteonecrosis of the talus. J Bone Joint Surg Am. 2015; 97(16): 1348-1353.

11. Ruatti S, Corbet C, Boudissa M, et al. Total talar prosthesis replacement after talar extrusion. J Foot Ankle Surg. 2017;56(4):905-909.

12. Gadkari KP, Anderson JG, Bohay DR, Maskill JD, Padley MA, Behrend LA. An eleven-year follow-up of a custom talar prosthesis after open talar extrusion in an adolescent patient: a case report. JBJS Case Connect. 2013;3(4):e118-e118.

13. Tsukamoto S, Tanaka Y, Maegawa N, et al. Total talar replacement following collapse of the talar body as a complication of total ankle arthroplasty: a case report. J Bone Joint Surg Am. 2010;92(11):2115-2120.

14. Angthong C. Anatomic total talar prosthesis replacement surgery and ankle arthroplasty: an early case series in Thailand. Orthop Rev. 2014; 6(3):5486. 
15. Ando Y, Yasui T, Isawa K, Tanaka S, Tanaka Y, Takakura Y. Total talar replacement for idiopathic necrosis of the talus: a case report. J Foot Ankle Surg. 2016;55(6):1292-1296.

16. Stevens BW, Dolan CM, Anderson JG, Bukrey CD. Custom talar prosthesis after open talar extrusion in a pediatric patient. Foot Ankle Int. 2007;28(8):933-938.

17. Tracey J, Arora D, Gross CE, Parekh SG. Custom 3D-printed total talar prostheses restore normal joint anatomy throughout the hindfoot. Foot Ankle Spec. Epub 2018 Mar 1.

18. Wong KC, Kumta SM, Geel NV, Demol J. One-step reconstruction with a 3D-printed, biomechanically evaluated custom implant after complex pelvic tumor resection. Comput Aided Surg. 2015;20(1):14-23.

19. Xu N, Wei F, Liu X, et al. Reconstruction of the upper cervical spine using a personalized 3d-printed vertebral body in an adolescent with ewing sarcoma. Spine. 2016;41(1):E50-E54.

20. Lu M, Min L, Xiao C, et al. Uncemented three-dimensional-printed prosthetic replacement for giant cell tumor of distal radius: a new design of prosthesis and surgical techniques. Cancer Manag Res. 2018; 10:265-277.

21. Lipińska J, Kutwin L, Wawrzycki M, Olbrzymek L, Jabłoński S. Chest reconstruction using a custom-designed polyethylene 3D implant after resection of the sternal manubrium. Onco Targets Ther. 2017;10: 4099-4103.

22. Kurtz SM, Ong K. Contemporary total hip arthroplasty: alternative bearings. In: Kurtz SM, editor. UHMWPE Biomaterials Handbook: Ultra High Molecular Weight Polyethylene in Total Joint Replacement and Medical Devices. 3rd ed. Oxford: William Andrew Publishing/ Elsevier; 2016:72-105.
23. Affatato $\mathrm{S}$. The history of biomaterials used in total hip arthroplasty (THA). In: Perspectives in Total Hip Arthroplasty: Advances in Biomaterials and their Tribological Interactions. Sawston: Woodhead Publishing; 2014:19-36.

24. Martinelli N, Bianchi A, Martinkevich P, et al. Return to sport activities after subtalar arthroereisis for correction of pediatric flexible flatfoot. J Pediatr Orthop B. 2018;27(1):82-87.

25. Needleman RL. A surgical approach for flexible flatfeet in adults including a subtalar arthroereisis with the MBA sinus tarsi implant. Foot Ankle Int. 2006;27(1):9-18.

26. Saxena A, Via AG, Maffulli N, Chiu H. Subtalar arthroereisis implant removal in adults: a prospective study of 100 patients. J Foot Ankle Surg. 2016;55(3):500-503.

27. Yavuz U, Sökücü S, Demir B, Özer D, Özcan C, Kabukçuoğlu YS. Isolated subtalar fusion for neglected painful intra-articular calcaneal fractures. Acta Orthop Traumatol Turc. 2014;48(5):541-545.

28. Ebalard M, Le Henaff G, Sigonney G, et al. Risk of osteoarthritis secondary to partial or total arthrodesis of the subtalar and midtarsal joints after a minimum follow-up of 10 years. Orthop Traumatol Surg Res. 2014;100(4 Suppl):S231-S237.
Therapeutics and Clinical Risk Management

\section{Publish your work in this journal}

Therapeutics and Clinical Risk Management is an international, peerreviewed journal of clinical therapeutics and risk management, focusing on concise rapid reporting of clinical studies in all therapeutic areas, outcomes, safety, and programs for the effective, safe, and sustained use of medicines. This journal is indexed on PubMed Central, CAS,

\section{Dovepress}

EMBase, Scopus and the Elsevier Bibliographic databases. The manuscript management system is completely online and includes a very quick and fair peer-review system, which is all easy to use. Visit http://www.dovepress.com/testimonials.php to read real quotes from published authors.

Submit your manuscript here: http://www.dovepress.com/therapeutics-and-clinical-risk-management-journal 